\title{
GF-3 SAR IMAGE DESPECKLING BASED ON THE IMPROVED NON-LOCAL MEANS USING NON-SUBSAMPLED SHEARLET TRANSFORM
}

\author{
Rui Shi ${ }^{1}$, Zengguo Sun ${ }^{2, *}$ \\ ${ }^{1}$ School of Computer Science, Shaanxi Normal University, Xi'an China, 1424022567@qq.com \\ ${ }^{2}$ School of Computer Science, Shaanxi Normal University, Xi'an China, duffer2000@163.com
}

KEY WORDS: GF-3 SAR, non-subsampled Shearlet transform, image despeckling, improved Non-Local Means

\begin{abstract}
:
GF-3 synthetic aperture radar (SAR) images are rich in information and have obvious sparse features. However, the speckle appears in the GF-3 SAR images due to the coherent imaging system and it hinders the interpretation of images seriously. Recently, Shearlet is applied to the image processing with its best sparse representation. A new Shearlet-transform-based method is proposed in this paper based on the improved non-local means. Firstly, the logarithmic operation and the non-subsampled Shearlet transformation are applied to the GF-3 SAR image. Secondly, in order to solve the problems that the image details are smoothed overly and the weight distribution is affected by the speckle, a new non-local means is used for the transformed high frequency coefficient. Thirdly, the Shearlet reconstruction is carried out. Finally, the final filtered image is obtained by an exponential operation. Experimental results demonstrate that, compared with other despeckling methods, the proposed method can suppress the speckle effectively in homogeneous regions and has better capability of edge preserving.
\end{abstract}

\section{INTRODUCTION}

GF-3 satellite was successfully launched in August 10, 2016 at the Taiyuan satellite launch center. It is meaningful for the country's economic development, the security of the country and people's safety [1]. However, due to the coherent imaging system, the speckle influences the understanding and the interpretation of the GF-3 SAR images. Therefore, the processing of the speckle suppression is a big challenge for the GF-3 SAR images [2]. The GF-3 SAR images have the better sparsity with the obvious edge, texture and point information. Only considering the sparse characteristics can choose the best methods about the image denoising. Two mainly methods about image denoising are adopted. One is the spatial filtering that only considers the statistical characteristics of sliding windows and never considers the sparsity of the images such as the Lee filter [3,4], Kuan filter [5,6], and the other is the frequency domain filtering that makes some transformation to the image [7], such as the wavelet hard threshold, wavelet soft threshold. The wavelet analysis can not satisfy the demand of the highresolution SAR images in image denoising. In recent years, the multiscale geometric analysis (MGA) has widely used for the speckle suppression such as Ridgelet transform, Curvelet transform, Contourlet transform and Shearlet transform [8]. Shearlet is used for images despeckling with its optimal sparse representation and the complete mathematical theory, it can effectively deal with the high-resolution SAR images that have the obvious sparse features [9]. In this paper a new method about the GF-3 SAR images is presented based on the nonsubsampled Shearlet transforms and the improved non-local means. The non-subsampled operation is adopted because the sampling operation will cause the spectrum aliasing $[10,11,12]$. The improved non-local means can deal with problems that the image details are smoothed overly and the weight distribution is insecure $[13,14]$.

\section{GF-3 SAR IMAGE}

GF-3 satellite has the 12 imaging modes such as the Spotlight, Strip, Scan, Wave, High and Low incidence angle and so on and these modes can switch freely. GF-3 satellite with 1-meter resolution imaging mode has become the highest resolution satellite system in the world with the C-band multi-polarized satellite. It can provide users with the long-time stable data support services [15]. Fig.1 is a scene of city SAR image that is obtained by the GF-3 satellite based on the satellite parameters, image processing and relative radiometric correction. Table 1 is the parameters of the above image.

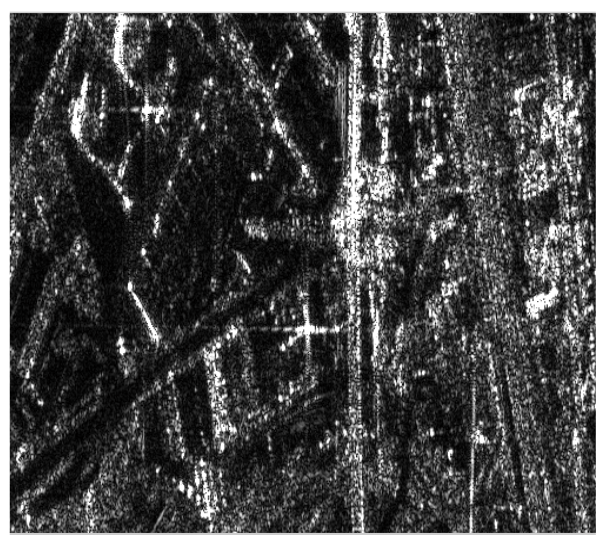

Fig 1. GF-3 SAR image 


\begin{tabular}{|c|c|c|c|c|}
\hline No. & Imaging model & Polarization & Resolution & Imaging position \\
\hline Fig.1 & FSI & HH & $5 \mathrm{~m}$ & $\begin{array}{c}\text { E113.4 } \\
\text { N34.7 }\end{array}$ \\
\hline
\end{tabular}

Table 1. The parameters of GF-3 SAR image

\section{ALGORITHM DESCRIPTION}

\subsection{Shearlet Transform}

1. The concept of the Shearlet

K.Guo and G.Easley proposed a new affine system that combined the multiscale analysis with the geometric. When dimension $n=2$, the affine system with compound expansion is defined as [16]:

$$
\Psi_{A B}(\varphi)=\left\{\varphi_{j, l, k}(x)=|\operatorname{det} A|^{j / 2} \varphi\left(B^{l} A^{j} x-k\right): j, l \in Z, k \in Z^{2}\right\}
$$

Where $\varphi \in L^{2}\left(R^{2}\right), A, B$ are $2 \times 2$ invertible matrices, $j, l, k$ are scale, shear and translation parameters. $A=A_{0}=\left(\begin{array}{ll}4 & 0 \\ 0 & 2\end{array}\right)$ is the anisotropic expansion matrix, $B=B_{0}=\left(\begin{array}{ll}1 & 1 \\ 0 & 1\end{array}\right)$ is shear matrix.

2. The good local characteristics of the Shearlet [17]

The function $\left\{\hat{\varphi}^{(0)}\left(\varepsilon A_{0}^{-j} B_{0}^{-l}\right)\right\}$ is a split of the $D_{0}$ (Shown in Fig 1(a)).

$$
D_{0}=\left\{\left(\varepsilon_{1}, \varepsilon_{2} \in \hat{R}^{2}:\left|\varepsilon_{1} \geq 1 / 8\right|\right),\left|\varepsilon_{2} / \varepsilon_{1} \leq 1\right|\right\}
$$

$\sum_{j \geq 0} \sum_{l=-2^{j}}^{2^{j}-1}\left|\hat{\varphi}^{(0)}\left(\varepsilon A_{0}^{-j} B_{0}^{-l}\right)\right|^{2}=\sum_{j \geq 0} \sum_{l=-2^{j}}^{2^{j}-1}\left|\hat{\varphi}_{1}\left(2^{-2 j} \varepsilon_{1}\right)\right|^{2}\left|\hat{\varphi}_{2}\left(2^{j} \frac{\varepsilon_{2}}{\varepsilon_{1}}-l\right)\right|^{2}=1$

Where $\left(\varepsilon_{1}, \varepsilon_{2}\right) \in D_{0}$. It means that Shearlet is compactly supported in the frequency domain and has very good local characteristics.

3. The strong directional sensitivity of the Shearlet

The function $\varphi_{j, l, k}$ has the following frequency domain support:

$$
\sup p \hat{\varphi}_{j, l, k}^{0} \subset\left\{\left(\varepsilon_{1}, \varepsilon_{2}\right): \varepsilon_{1} \in\left[-2^{2 j-1},-2^{2 j-4}\right] \cup\left[2^{2 j-4}, 2^{2 j-1}\right],\left|l 2^{-j}+\varepsilon_{2} / \varepsilon_{1}\right| \leq 2^{-j}\right\}
$$

It means that each element $\hat{\varphi}_{j, l, k}$ is supported by the trapezoid whose size is $2^{2 j} \times 2^{j}$, and the direction is along with the line with the slope $l 2^{-j}$ (Shown in Fig 1(b)). It should be noted that the Shearlet has strong directional sensitivity.

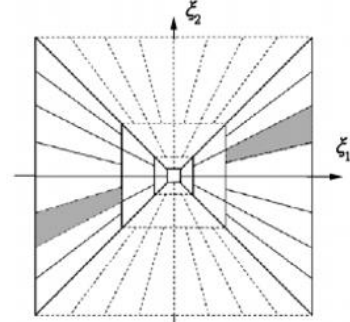

(a)

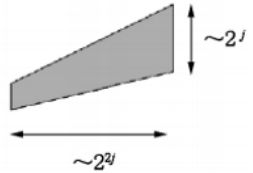

(b)
Fig 2. Spatial-frequency plane and frequency support of Shearlets: (a) Frequency domain split of Shearlet, (b) Frequency domain support of Shearlet.

4. The optimal sparse representation of the Shearlet

Denoting $f$ as the function that the direction is $C^{2}$ except a curve that is piecewise $C^{2}$ continuous respectively. $f_{N}^{S}$ is the approximation of the maximum Shearlet coefficient that the count is $N$ of $f$. The relationship between them is:

$$
\left\|f-f_{N}^{s}\right\|_{2}^{2} \leq C N^{-2}(\log N)^{3}
$$

The approximation order of the Shearlet can be reached $O\left(N^{-2}(\log N)^{3}\right)$ and the Shearlet can present the images that have rich information optimally.

\subsection{Non-Subsampled Shearlet Transform}

The non-subsampled Shearlet transform can be divided into 3 steps. Firstly, Since the Laplacian Pyramid decomposition includes the down sampling operation. According to the multisampling rate theory, the down sampling operation may lead to the aliasing in the filtered image. So the non-subsampled Laplacian Pyramid method is applied to the GF-3 SAR image splitting the image into the low-pass filtered image and the high-pass filtered image. Secondly, the Fourier transform is used in pseudo polar grid to deal with the high-pass filtered image. Lastly, the Shearlet coefficient is obtained by rearranging the data into the Cartesian coordinate system using the band-pass filtering and inverse Fourier transform [18].

\subsection{Improved non-local means}

Denoting $v=\{v(i), i \in I\}$ as a scene of SAR image that contains the speckle respectively, the non-local means is given by :

$$
N L[v](i)=\frac{\sum_{j \in I} f(i, j) v(j)}{\sum_{j \in I} f(i, j)}
$$


Where $i, j$ are the pixel of the image, $I$ is the image domain, $f$ is the kernel function.

A new kernel function is used in non-local means. The function is given by $[19,20]$

$$
f(i, j)=\frac{1}{\sqrt{1+(d(i, j) / h)^{2 N}}}
$$

Where $h$ is the smoothing parameter and determines the decay rate of the filter function, $N$ is the filter parameter, $d(i, j)$ uses the Euclidean distance as the similarity of each adjacency matrix. The $d(i, j)$ is given by:

$$
d(i, j)=\left\|v\left(N_{i}\right)-v\left(N_{j}\right)\right\|_{2, \alpha}^{2}
$$

Where $i, j$ are the core of the square neighborhood $N_{i}$, $N_{j} . \alpha$ is the standard deviation of the Gauss weighted and $\alpha>0,\|.\|_{2}$ is the norm.

\subsection{GF-3 SAR image despeckling using the non-subsampled} Shearlet transform based on the improved Non-Local means.

Step 1: The logarithmic transform is made to the GF-3 SAR image.

Step 2: The non-subsampled Shearlet transform is applied to the image attained by the Step 1 to get low and the high frequency coefficients.

Step 3: The improved non-local means is made to the high frequency coefficients.

Step 4: Making the Shearlet reconstruction to the low and the high frequency coefficients by the invert nonsubsampled Shearlet transform.

Step 5: The de-noised image is obtained by making an exponential operation.

The flowchart of the proposed method based on the nonsubsampled Shearlet transform and the improved non-local means is shown in Fig 3.

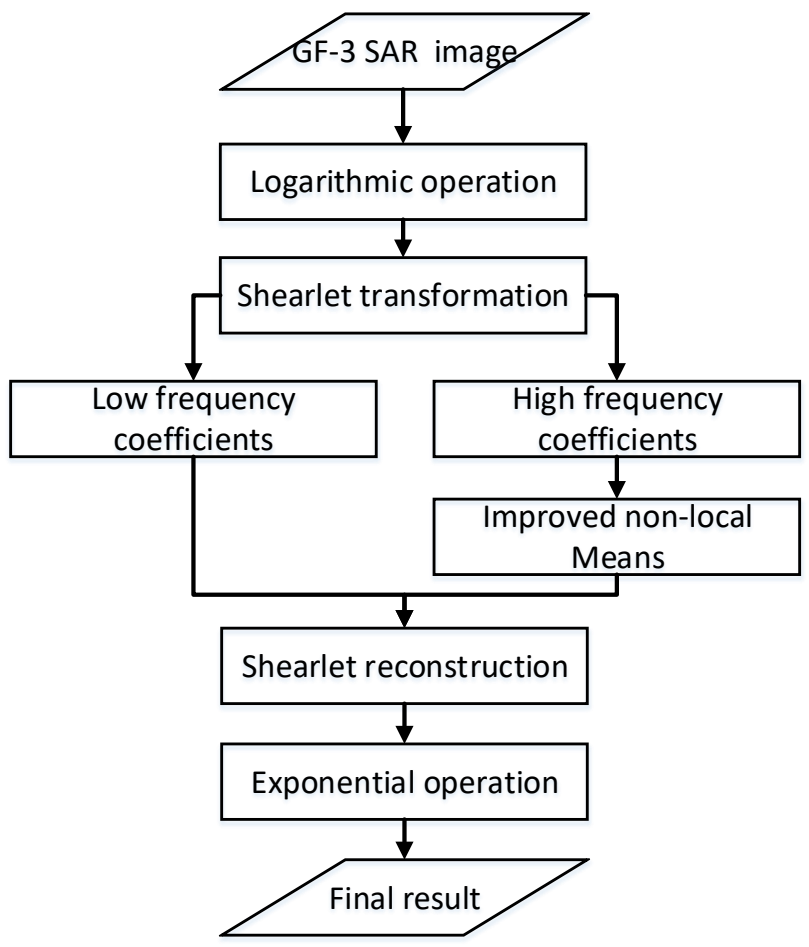

Fig 3. The flowchart of our method

\section{DESPECKLING EXPERIMENT}

Fig 4 shows the despeckling results of experimental data. It can be seen that the Lee filter, Gamma MAP filter cannot smooth the speckle effectively. Although the improved non-local means can smooth the speckle effectively, the edge information of the image is seriously blurred. However, our method can suppress the speckle effectively in homogeneous region and preserve the edge information wonderfully.

The quantitative index ENL and DCV are used to evaluate the capability of suppressing the speckle and preserving the edge information. ENL is the evaluation index of the de-noised results of the homogeneous region. Higher ENL means better smoothing ability [21]. DCV is the evaluation index of the capability of preserving edge information. It is defined as the difference of coefficient of variation between the denoised image and the original image. If the value is closer to 0 , the capability of preserving the edge information is better.

Table 2 is the quantitative index. It can be seen that compared with other despeckling methods, the proposed method can suppress the speckle effectively in homogeneous regions and it has better capability of edge preserving.

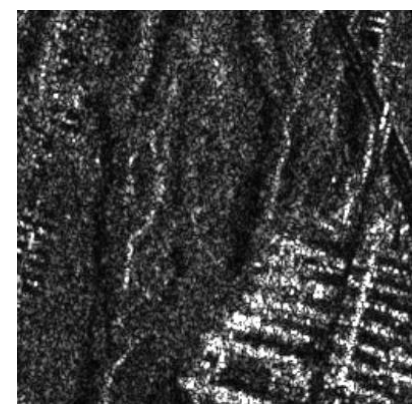

(a) 


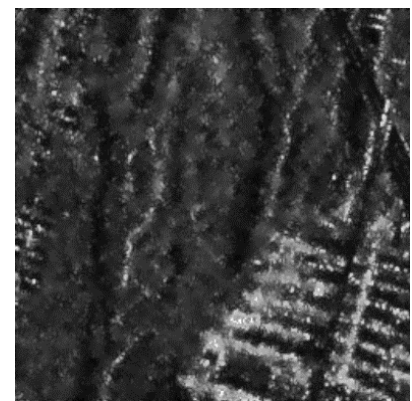

(b)

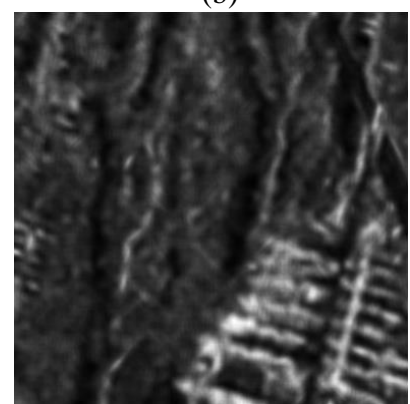

(d)

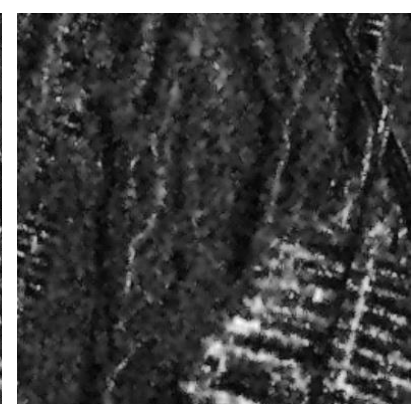

(c)

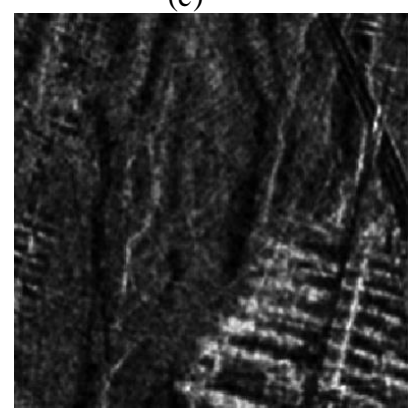

(e)

Fig 4. Despeckling results: (a) original image, (b) Lee filter, (c) Gamma MAP filter, (d) improved non-local means, (e) our method.

\begin{tabular}{|c|c|c|c|c|}
\hline & Lee filter & $\begin{array}{c}\text { Gamma } \\
\text { MAP filter }\end{array}$ & $\begin{array}{c}\text { improved } \\
\text { non-local } \\
\text { means }\end{array}$ & $\begin{array}{c}\text { our } \\
\text { method }\end{array}$ \\
\hline ENL & 3.1678 & 2.9481 & 5.2748 & 9.6291 \\
\hline DCV & 0.0320 & 0.0252 & 0.1515 & 0.0029 \\
\hline
\end{tabular}

Table 2. Quantitative index

Fig 5 shows the despeckling results of experimental data. It can be seen that our method can smooth the speckle effectively and preserve the edge information wonderfully compared with other methods.

Fig 6 is the speckle pattern of the de-noised image. The speckle pattern is defined by the ratio of the observed image and the denoised image. If there are obvious granular speckle in the speckle pattern, the capability of smoothing speckle is better. If there is very little structure information in the speckle pattern, the capability of preserving edges is higher [22]. It can be seen that our method can smooth the speckle and preserve the edge information effectively.

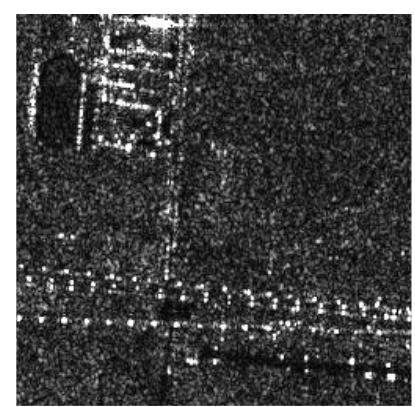

(a)



(b)

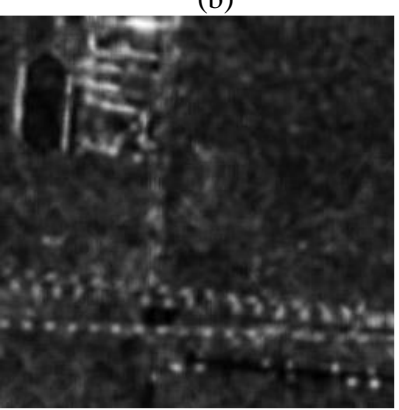

(d)

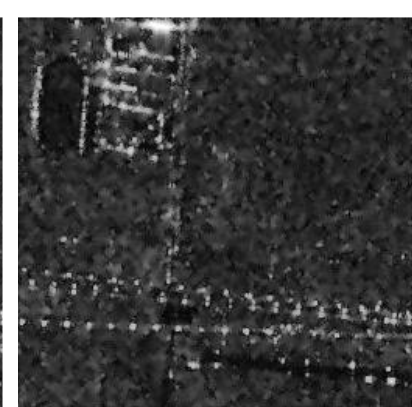

(c)

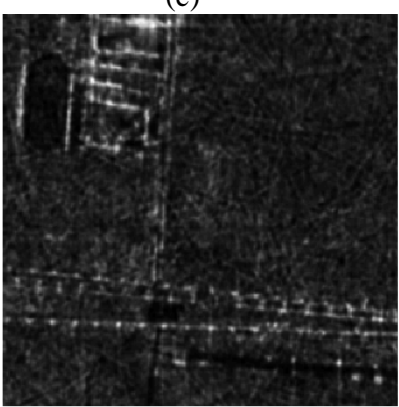

(e)
Fig 5. Despeckling results: (a) original image, (b) Lee filter, (c) Gamma MAP filter, (d) improved non-local means, (e) our method.

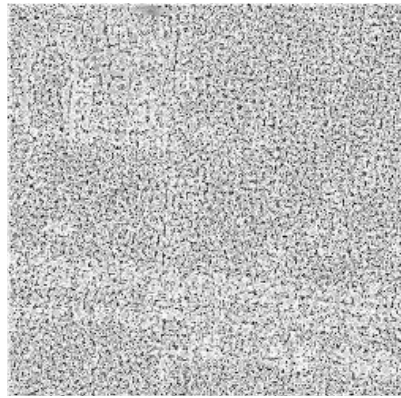

(a)

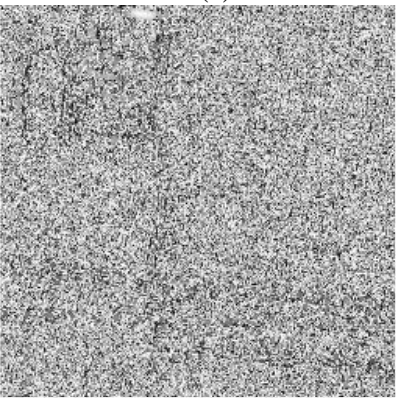

(c)

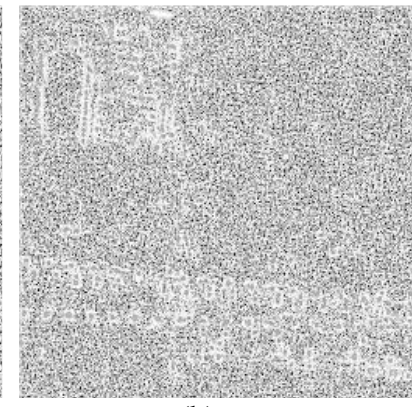

(b)

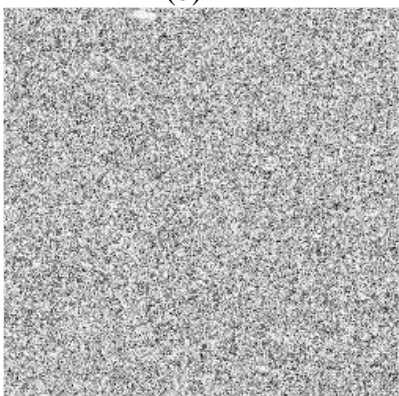

(d)
Fig 6. Speckle pattern: (a) Lee filter, (b) Gamma MAP filter, (c) improved non-local means, (d) our method.

\section{CONCLUSION}

In order to suppress the speckle in GF-3 SAR images, the nonsubsampled Shearlet transform is proposed based on the improved non-local means using non-subsampled Shearlet transform in this paper. The non-subsampled Shearlet transform can deal with the GF-3 SAR images that have the better sparsity. The improved non-local means is used for solving the problems that the image details are suppressed overly. Despeckling experiments demonstrate the efficiency of the proposed filter, leading to a better trade-off between speckle suppression and edge preservation. 


\section{REFERENCES}

Shao W, Sheng Y, Sun J. Preliminary Assessment of Wind and Wave Retrieval from Chinese Gaofen-3 SAR Imagery.[J]. Sensors, 2017, 17(8): 1-13.

Ravi A, Giriprasad M N, Naganjaneyulu P V. SAR images denoising using a novel stochastic diffusion wavelet scheme[J]. Cluster Computing, 2017(8): 1-9.

Yommy A S, Liu R, Onuh S O, et al. SAR image despeckling and compression using K-nearest neighbour based lee filter and wavelet[C]// International Congress on Image and Signal Processing. IEEE, 2016: 158-167.

Lee J.S. Digital image enhancement and noise filtering by use of local statistics. IEEE Trans. Pattern Anal. Machine Intell. 1980, Mar, 2(2) 165-168.

Sharma A, Bhateja V, Tripathi A. An Improved Kuan Algorithm for Despeckling of SAR Images[M]// Information Systems Design and Intelligent Applications. Springer India, 2016: 663-672.

Kuan D.T, Sawchuk A.A and Strand T.C. Adaptive noise smoothing filter for image with signal-dependent noise. IEEE Trans. Pattern Anal. Mach. Intell 1985 Mar, 7(2): 165-177.

Aravind B N, Suresh K V. An improved image denoising using wavelet transform[C]// International Conference on Trends in Automation, Communications and Computing Technology. IEEE, 2016: 1-5.

Kutyniok G, Labate D. Shearlets: Multiscale Analysis for Multivariate Data[M]. Birkhäuser Basel, 2012.

Liu S, Liu M, Li P, et al. SAR Image Denoising via Sparse Representation in Shearlet Domain Based on Continuous Cycle Spinning[J]. IEEE Transactions on Geoscience \& Remote Sensing, 2017(99): 1-8.

Liu S, Geng $\mathrm{P}$, Shi $\mathrm{M}$, et al. SAR Image De-noising Based on Generalized Non-local Means in Non-subsample Shearlet Domain[M]// Proceedings of the 2015 International Conference on Communications, Signal Processing, and Systems. Springer Berlin Heidelberg, 2016.

Hu S, Ma X, Liu S, et al. SAR Image De-noising Based on Non-Loca Similar Block Matching in NSST Domain[C]// IEEE, International Conference on Signal Processing. IEEE, 2017: 832-836.

Gao G. Image Denoising by Non-Subsampled Shearlet Domain Multivariate Model and Its Method Noise Thresholding[J]. Optik International Journal for Light and Electron Optics, 2013, 124(22): 5756-5760.

Zhong H, Yang C, Zhang X. A New Weight for Nonlocal Means Denoising Using Method Noise[J]. IEEE Signal Processing Letters, 2012, 19(8): 535-538.

Zhang S, Jing H. Fast Log-Gabor-Based Nonlocal Neans Image Denoising Methods[C]// IEEE International Conference on Image Processing. IEEE, 2015: 2724-2728.

Xiang Y, Wang F, You H. An Automatic and Novel SAR Image Registration Algorithm: A Case Study of the Chinese GF-3 Satellite[J]. Sensors, 2018, 18(2): 672.
G. Easley, D. Labate, W.Q. Lim. Sparse directional image representation using the discrete Shearlets transform [J]. Applied and Computational Harmonic Analysis, 2008, 25(1): 25-46.

Lim W Q. The discrete shearlet transform: a new directional transform and compactly supported shearlet frames.[J]. IEEE Transactions on Image Processing A Publication of the IEEE Signal Processing Society, 2010, 19(5): 1166-1180.

Hu S, Ma X, Liu S, et al. SAR image de-noising based on non-local similar block matching in NSST domain[C]// IEEE, International Conference on Signal Processing. IEEE, 2017: 832-836.

Tian J, Yu W Y, Xie S L. On the kernel function selection of nonlocal filtering for image denoising[C]// International Conference on Machine Learning and Cybernetics. IEEE, 2008: 2964-2969.

Lai R, Dou X X. Improved non-local means filtering algorithm for image denoising[C]// International Congress on Image and Signal Processing. IEEE, 2010: 720-722.

Parrilli S, Poderico M, Angelino C V, et al. A Nonlocal SAR Image Denoising Algorithm Based on LLMMSE Wavelet Shrinkage[J]. IEEE Transactions on Geoscience \& Remote Sensing, 2012, 50(2): 606-616.

Hou $\mathrm{B}$, Zhang $\mathrm{X}, \mathrm{Bu} \mathrm{X}$, et al. SAR Image Despeckling Based on Nonsubsampled Shearlet Transform[J]. IEEE Journal of Selected Topics in Applied Earth Observations \& Remote Sensing, 2012, 5(3): 809-823. 